\title{
Additive effects of atorvastatin combined with sitagliptin on rats with myocardial infarction: a pilot study
}

Xiao Keㄹ ${ }^{1}$ Bin $\mathrm{Ke}^{2}$, Xing Wang ${ }^{2}$, Shaoyun $\mathrm{Wu}^{2}$, Rongfeng Yang ${ }^{2}$, Chengheng $\mathrm{Hu}^{2}$

${ }^{1}$ Department of Cardiology, Shenzhen Sun Yat-sen Cardiovascular Hospital, Shenzhen, Guangdong, China

${ }^{2}$ Department of Cardiology, the First Affiliated Hospital of Sun Yat-sen University, Guangzhou, China

Submitted: 27 October 2016

Accepted: 28 November 2016

Arch Med Sci 2017; 13, 4: 956-961

DOI: https://doi.org/10.5114/aoms.2017.68143

Copyright @ 2017 Termedia \& Banach

\section{Abstract}

Introduction: Atorvastatin and sitagliptin are able to exert cardio-protective effects. However, whether atorvastatin plus sitagliptin could confer additive benefits for rats with myocardial infarction (MI) is unknown.

Material and methods: Forty rats with $\mathrm{MI}$ were produced and 37 surviving rats were randomly divided into atorvastatin $(10 \mathrm{mg} / \mathrm{kg}$ daily, $n=9)$, sitagliptin (10 mg/kg daily, $n=9)$, combined (10 mg/kg daily atorvastatin plus $10 \mathrm{mg} / \mathrm{kg}$ daily sitagliptin, $n=9$ ), and control groups ( $3 \mathrm{ml}$ normal saline daily, $n=10$ ). Fourteen days later, cardiac function was detected and fasting venous blood was sampled for lipid profiles and glucose evaluation. Cardiac tissues were used for hematoxylin-eosin staining, for interleukin-6 (IL-6) and tumor necrotic factor- $\alpha$ (TNF- $\alpha$ ) evaluation, and for rho-associated kinase 2 (ROCK2) assessment.

Results: Fourteen days after MI, the inflammatory reaction regarding the degree of leukocyte infiltration and IL- 6 and TNF- $\alpha$ expression in cardiac tissues was ameliorated in atorvastatin and sitagliptin groups compared to the control group $(p<0.05)$. In addition, ROCK 2 was attenuated by either atorvastatin or sitagliptin $(p<0.05)$. Echocardiography showed that cardiac function was significantly improved with atorvastatin and sitagliptin therapy $(p<0.05)$. Overall, all these benefits were further enhanced by combined therapy, suggesting that atorvastatin combined with sitagliptin therapy has additive effects on reducing cardiac inflammation and improving cardiac function. No significant changes in lipid profiles or glucose were observed, suggesting that the benefits derived from atorvastatin and sitagliptin therapy might not depend on cholesterol and glucose modulation.

Conclusions: In rats with $\mathrm{Ml}$, atorvastatin plus sitagliptin therapy provides additive effects for cardio-protection, and mechanisms operating in these processes may be due to ROCK2 diminishment.

Key words: atorvastatin, sitagliptin, myocardial infarction, mechanism.

\section{Introduction}

Atherosclerotic cardiovascular disease (ASCVD), especially myocardial infarction (MI), is the leading cause of morbidity and mortality around the world at present [1]. Statins, 3-hydroxy-methylglutaryl-coenzyme A (HMG-CoA) reductase inhibitors, are a class of medications for cholesterol modification and have been broadly used for the primary and secondary prevention of ASCVD [2-4]. Previously, many experimental and

\author{
Corresponding author: \\ Chengheng $\mathrm{Hu}$ \\ Department of Cardiology \\ The First Affiliated Hospital \\ of Sun Yat-sen University \\ 58 Zhongshan Road 2 \\ Guangzhou 510080, China \\ Phone: +86 2087755766 , \\ ext. 8164 \\ Fax: +862087755766 \\ ext. 8756 \\ E-mail: \\ huchengheng138@163.com
}


clinical studies have consistently demonstrated that statin therapy is beneficial for ameliorating inflammation and improving cardiac function after $\mathrm{MI}$, and the mechanisms operating in these processes are ascribed to statins' effects on inhibiting rho-associated kinase (ROCK) and associated signaling pathways $[5,6]$.

In the past decade, several studies have revealed that dipeptidyl peptidase-4 (DPP-4) inhibitor has additional cardio-protective effects other than regulating glucose metabolism, and these benefits were dependent on its anti-inflammatory and endothelium-protection efficacy [7-9]. In the last decade, with increasing prevalence of diabetes mellitus, a major risk factor of ASCVD, DPP-4 inhibitor has been broadly used in clinical practice for its potential cardiac protection. In addition, since the risk of diabetes is equivalent to that of ASCVD, statins have also been recommended for diabetic populations for secondary prevention. It is therefore interesting and clinically relevant to investigate whether statins combined with DPP-4 inhibitor could provide additive benefit for the cardiovascular system. Theoretically, statins combined with DPP-4 inhibitor therapy should have better cardio-protective efficacies owing to their potent pleiotropic effects on vascular systems. Nevertheless, the data are scant and the underlying mechanisms are also not well studied. In this study we conducted basic research to address these issues, and we considered that the preliminary data from our present research would shed light on the clinical preferred application of statins combined with DPP-4 inhibitors in patients with high cardiovascular risk.

\section{Material and methods}

\section{Animals and protocol}

Forty healthy male Sprague-Dawley rats weighing 210-230 g were obtained from the Experimental Animal Center of Guangdong Province, China. The study was approved by the Ethic Committee of Sun Yat-sen University. All animals received humane care in compliance with the Guide for the Care and Use of Laboratory Animals of the Institute of Laboratory Animal Resources, National Research Council.

After 1 week of accommodation, 40 rats underwent $\mathrm{MI}$ model establishment. Briefly, induction of MI was performed as previously described by permanent ligation of the left anterior descending coronary artery with the help of ventilation (tidal volume, $6.0 \mathrm{ml}$; respiratory rate, 70 cycles $/ \mathrm{min}$ ) [10]. Thereafter, the surviving 37 rats were randomly divided into 4 groups as follows: atorvastatin group (10 mg/kg daily, oral gavage, $n=9)$, sitagliptin group (10 mg/kg daily, oral gavage, $n=9$ ), combined group (10 mg/kg daily atorvastatin plus $10 \mathrm{mg} / \mathrm{kg}$ daily sitagliptin, $n=9$ ), and control group (3 $\mathrm{ml}$ of normal saline daily, oral gavage, $n=10)$. The medications were prescribed $24 \mathrm{~h}$ after MI was produced and the therapeutic duration was 2 weeks.

\section{Cardiac function measurement with echocardiography}

Briefly, 2 weeks later, cardiac function in terms of left ventricular ejection fraction (LVEF), fractional shortening (FS), left ventricular end-systolic and end-diastolic diameter (LVDs and LVDd) were measured by echocardiography (ATL-HDI500 equipped with a $10-\mathrm{MHz}$ image transducer). Briefly, LVEF was calculated by $\left(\mathrm{LVDd}^{2}-\mathrm{LVDs}^{2}\right) / \mathrm{LVDs}^{2}$. Before performance, all the rats were under anesthesia and all procedures were performed by an experienced investigator who was blinded to these groups. Assessment was conducted three times to obtain the average values of each cardiac function parameter.

\section{Hematoxylin-eosin staining of cardiac tissue}

After cardiac function was measured, all animals were humanely sacrificed and cardiac tissues were embedded in $4 \mu \mathrm{m}$ paraffin sections for hematoxylin-eosin (HE) staining. The extent of leukocyte infiltration was evaluated by counting the number of leukocytes in 10 randomly peri-infarcted areas of each group at 200× magnification (Olympus BX51 microscope, Tokyo, Japan). All the evaluations were performed by an investigator who was blinded to the assignment group.

\section{Evaluation of inflammatory cytokines by western blot}

Expression of inflammatory cytokines including interleukin-6 (IL-6) and tumor necrotic factor- $\alpha$ (TNF- $\alpha$ ) in cardiac tissues was evaluated by western blot. Tissues were extracted from myocardium and then homogenized in $500 \mu \mathrm{l}$ of lysate. After electrophoresis, trans-membrane and blocking, membrane was incubated at $4^{\circ} \mathrm{C}$ overnight with rabbit anti-rat IL-6 (Sigma, St. Louis, MO, 15018, SAB4300383, 1 : 1500) or TNF- $\alpha$ (Cell Signaling Technology, Danvers, MA, \#3727, 1 : 1500). The membranes were washed 3 times with TBS-T and incubated with goat anti-rabbit IgG HRP-conjugated secondary antibody (Cell Signaling Technology, Danvers, MA, \#7074, $1: 4000)$ at room temperature for 1 h. Protein of interest was detected by chemiluminescence, and optical density (OD) was measured in grey scale images with the Volume Contour method. Glyceraldehyde-phosphate dehydrogenase (GAPDH) (Cell Signaling Technology, Danvers, MA, \#2118, 
1 : 8000) was used as a loading control. All the data were presented as relative expression after being normalized to GAPDH.

\section{ELISA assay for ROCK2 measurement}

Quantitative immunoassay was used for evaluating expression of ROCK 2 in cardiac tissues after 2 weeks of therapy. Procedures were performed in accordance with the manufacturer's protocol (Huamei Biology Company, Wuhan, China, CSBEL020059RA). The detected range is from 62.5 to $4000 \mathrm{pg} / \mathrm{ml}$, and the detected wavelength is $450 \mathrm{~nm}$. All the measurements were repeated 3 times to determine the arithmetic average.

\section{Evaluation of the changes of lipid profiles and glucose}

After 14 days of therapy, $8 \mathrm{ml}$ of fasting venous blood was sampled for lipid profiles and glucose detection by an Automatic Biochemistry Analyzer (Beckman Coulter UniCel DxC 800 Synchron).

\section{Statistical analysis}

All continuous variables were expressed as mean \pm SD, and analyses were performed with SPSS software, version 17.0 (SPSS Science, Chicago, IL, USA). Statistical significance between differ- ent groups was evaluated with ANOVA post-hoc tests, and a value of $p<0.05$ was considered to be statistically significant.

\section{Results}

\section{Comparison of anti-inflammatory effects between groups}

As shown in Figures 1 A, B, 14 days after MI, the inflammatory reaction in terms of the degree of leukocyte infiltration and IL- 6 and TNF- $\alpha$ expression in cardiac tissues was ameliorated in atorvastatin and sitagliptin groups when compared to the control group $(p<0.05)$. Notably, these effects were further enhanced in the combined group, strongly suggesting that atorvastatin combined with sitagliptin therapy has additive effects on reducing cardiac inflammation. The improvement of inflammation was better in the atorvastatin group than in the sitagliptin group, but no statistical significance was observed.

\section{Comparison of ROCK2 between groups}

After 14 days of therapy, ROCK2 in cardiac tissues was compared between groups. As presented in Figure 2, atorvastatin or sitagliptin therapy significantly reduced ROCK 2 when compared to the control group $(p<0.05)$, and this benefit was fur-

A

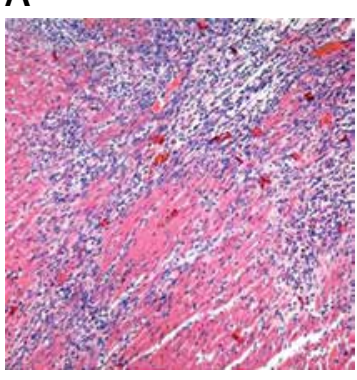

Controlled

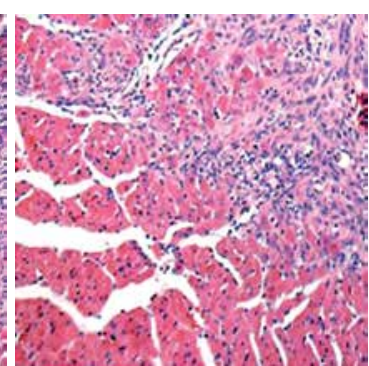

Sitagliptin

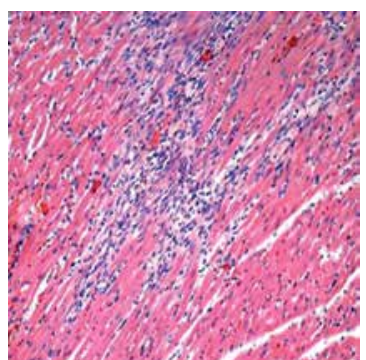

Atorvastatin

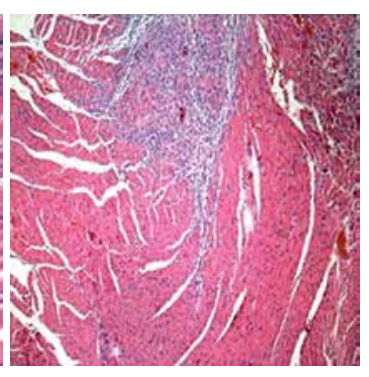

Combined

B
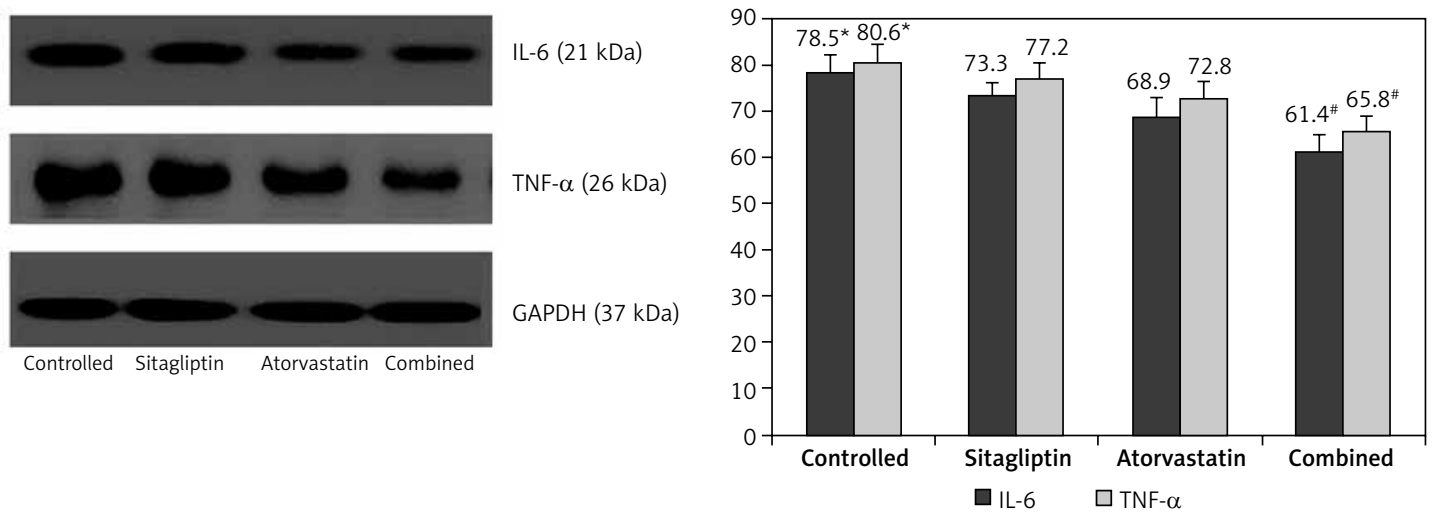

Figure 1. Evaluation of inflammatory parameters: A - shows the degree of leukocyte infiltration in cardiac tissues, B - shows the expressions of IL- 6 and TNF- $\alpha$ in cardiac tissues

${ }^{\star} P<0.05$ vs. other groups, ${ }^{*} p<0.05$ vs. sitagliptin and atorvastatin groups 
ther promoted by combined therapy, suggesting that atorvastatin combined with sitagliptin therapy had additive effects on diminishing ROCK2. No significant difference was observed between atorvastatin and sitagliptin groups, although it appeared that ROCK2 was modestly lower in the atorvastatin group.

\section{Comparison of cardiac function between groups}

After 14 days of therapy, cardiac function was evaluated by echocardiography and was compared between groups. As shown in Table I, atorvastatin or sitagliptin therapy significantly enhanced LVEF and fractional shortening (FS) when compared to the control group $(p<0.05)$. Moreover, LVDs and LVDd were both modestly reduced in atorvastatin and sitagliptin groups. Overall, cardiac function was further promoted by combined therapy $(p<0.05)$.

\section{Effects of atorvastatin and sitagliptin therapy on lipid profiles and glucose}

After 14 days of therapy, lipid profiles and fasting blood glucose were detected. As shown in Table II, no significant changes of lipid profiles or fasting blood glucose were observed between groups, suggesting that the effects of atorvastatin or sitagliptin therapy might be cholesterol and glucose independent.

\section{Discussion}

Data from our preliminary research revealed that in rats with $\mathrm{MI}, 14$ days of atorvastatin or

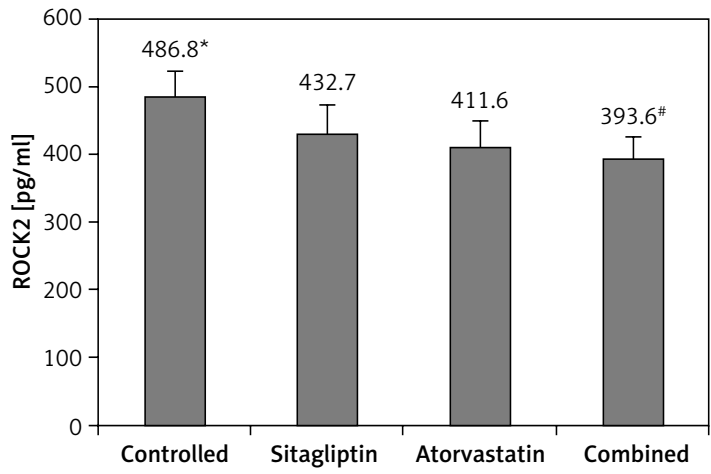

Figure 2. Comparison of ROCK2 between groups ${ }^{*} p<0.05$ vs. other groups, ${ }^{*} p<0.05$ vs. sitagliptin and atorvastatin groups.

sitagliptin therapy improves cardiac inflammation as reflected by reduced leukocyte infiltration, as well as diminished expression of IL- 6 and TNF- $\alpha$. In addition, ROCK2 is also decreased by either regimen as compared with the control group. Moreover, echocardiography shows that cardiac function in both atorvastatin and sitagliptin groups is significantly improved in comparison to the control group. Importantly, these benefits are further enhanced by combined therapy, strongly suggesting that atorvastatin plus sitagliptin therapy could provide additive benefits for improving cardiac inflammation and cardiac function after ischemic insult.

The inflammatory reaction after MI plays pivotal and adverse roles in cardiac remodeling [11]. Similarly, our current study also showed that in the control group, cardiac diastolic and systolic diameters were significantly elevated, as presented

Table I. Comparison of cardiac function between groups

\begin{tabular}{|c|c|c|c|c|}
\hline Parameter & Control & Sitagliptin & Atorvastatin & Combined \\
\hline LVEF (\%) & $47.5 \pm 6.6^{*}$ & $52.1 \pm 6.2$ & $55.3 \pm 5.6$ & $61.8 \pm 7.4^{\#}$ \\
\hline FS (\%) & $26.4 \pm 3.3^{*}$ & $30.5 \pm 3.8$ & $32.6 \pm 4.0$ & $36.4 \pm 3.0^{\#}$ \\
\hline LVDs [mm] & $41.6 \pm 4.5^{\star}$ & $37.4 \pm 3.8$ & $36.2 \pm 5.1$ & $33.9 \pm 4.6^{\#}$ \\
\hline LVDd [mm] & $32.7 \pm 3.3^{*}$ & $29.2 \pm 3.8$ & $29.3 \pm 2.8$ & $24.6 \pm 4.3^{\#}$ \\
\hline
\end{tabular}

${ }^{\star} P<0.05$ vs. other groups, ${ }^{\#} P<0.05$ vs. sitagliptin and atorvastatin groups.

Table II. Comparison of lipid profiles and glucose between each group

\begin{tabular}{|lcccc|}
\hline Parameter & Control & Sitagliptin & Atorvastatin & Combined \\
\hline TG $[\mathrm{mmol} / \mathrm{l}]$ & $1.5 \pm 0.6$ & $1.7 \pm 0.4$ & $1.7 \pm 0.6$ & $1.5 \pm 0.4$ \\
\hline TC $[\mathrm{mmol} / \mathrm{l}]$ & $4.3 \pm 0.3$ & $4.5 \pm 0.6$ & $4.6 \pm 0.5$ & $4.4 \pm 0.3$ \\
\hline HDL-C $[\mathrm{mmol} / \mathrm{l}]$ & $1.0 \pm 0.5$ & $1.0 \pm 0.3$ & $1.1 \pm 0.3$ & $1.2 \pm 0.4$ \\
\hline LDL-C $[\mathrm{mmol} / \mathrm{l}]$ & $2.7 \pm 0.5$ & $2.5 \pm 0.4$ & $2.4 \pm 2.3$ & $2.6 \pm 0.3$ \\
\hline FBG $[\mathrm{mmol} / \mathrm{l}]$ & $4.6 \pm 0.6$ & $4.8 \pm 0.5$ & $4.9 \pm 0.6$ & $4.6 \pm 0.4$ \\
\hline
\end{tabular}

TG - triglyceride, TC - total cholesterol, HDL-C - high-density lipoprotein cholesterol, LDL-C - low-density lipoprotein cholesterol, FBG - fasting blood glucose. 
in Table I. By mechanism, leukocytes infiltrate and accumulate in peri-infarcted areas and thereby elicit a cascade of inflammatory reactions which leads to cardiomyocytes' apoptosis and necrosis [12]. Previously, many studies have demonstrated that statin therapy could improve cardiac inflammation and reduce cardiomyocytes loss, and the mechanisms are associated with statins' pleiotropic effects $[13,14]$. For example, Cai et al. reported that in rats with acute myocardial infarction, atorvastatin therapy is beneficial for reducing inflammatory cytokine expression as well as decreasing leukocyte infiltration [10]. In addition, through improving endothelial function, statin therapy reduces leukocytes trans-endothelium migration, and the mechanism is associated with statins' effect on inhibiting ROCK [15]. Moreover, diminishing ROCK, statins promote nitric oxide generation and reduce reactive oxygen species production [16]. Consistent with previous reports, results from our present research also show that atorvastatin therapy could mitigate cardiac inflammation and reduce ROCK2. Owing to previous compelling evidence, we considered that inflammation improvement was at least partially ascribed to diminishment in ROCK2 with atorvastatin therapy. In addition, these improvements might in turn lead to improvement of cardiac remodeling as reflected by smaller systolic and diastolic diameters in rats with atorvastatin therapy than the control group, as shown in Table I.

Interestingly and importantly, similar improvement in cardiac inflammation and ROCK2 attenuation was also observed with sitagliptin therapy. However, these benefits were slightly less than that with atorvastatin therapy. Previously, some studies showed that other than regulating glucose metabolism, sitagliptin had a role in improving endothelial function and reducing inflammatory cytokine generation [17-19]. It might be the first mechanism by which sitagliptin reduced leukocyte infiltration and ameliorated inflammation. On the other hand, via an AMP-activated protein kinase (AMPK) and mitogen-activated protein kinase (MAPK)-dependent mechanism, sitagliptin reduces leukocyte-endothelium interaction and attenuates atherosclerosis progression in apo-lipoprotein-E knockout mice [20]. Therefore, we postulated that sitagliptin might ameliorate cardiac inflammation through the AMPK and MAPK signaling pathway, and the underlying mechanism might be associated with sitagliptin effects on diminishing ROCK2. Additionally, through increasing NO production and decreasing serum IL-6, C-reactive protein, and TNF- $\alpha$ levels $[9,21]$, sitagliptin should improve the general condition and thereby improve the cardiac microenvironment.
Notably, the above benefits of atorvastatin or sitagliptin therapy conferred to rats with $\mathrm{MI}$ were further enhanced by the combined therapy. With regard to the underlying mechanisms contributing to atorvastatin or sitagliptin therapy as mentioned above, we considered that the additive benefits with atorvastatin plus sitagliptin therapy might be at least partially dependent on their potent effects on reducing ROCK2, since no significant changes of lipid profiles and glucose were observed between different therapeutic groups. However, we should be cautious considering that the absence of effects on lipid reduction could be just due to the fact that the therapy duration was not long enough. Importantly, our present research additionally revealed that cardiac inflammation improvement and ROCK2 attenuation contributed to profound enhancement of cardiac function as reflected by the profound increase in LVEF and FS. Moreover, cardiac remodeling was also observed. Owing to the effect of statins in reducing cardiomyocyte apoptosis and the effect of sitagliptin in diminishing cardiac fibrosis, it was reasonable to conclude that atorvastatin plus sitagliptin therapy could provide additive benefits for preserving cardiac function.

Finally, with respect to the design of our present study, there are some limitations that should be addressed and, in addition, we suggest that the future directions should also be focused on these unaddressed issues. First of all, our current research was only a pilot study, and the uninvestigated mechanisms should be further studied. Second, as mentioned above, the AMPK and MAPK signaling pathway might be involved in the amelioration of the inflammatory reaction of sitagliptin therapy; however, our current study did not evaluate the AMPK and MAPK signaling pathway, and we suggest that future studies should evaluate these signaling pathways, which are critical for understanding the mechanisms of the pleiotropic effects of sitagliptin. Third, we did not assess the dose-dependent relationship of atorvastatin and sitagliptin therapy in rats with myocardial infarction, and it is essential if such a relationship could be corroborated regarding the strength of the findings' validity.

In conclusion, our present research suggests that in rats with $\mathrm{MI}$, atorvastatin plus sitagliptin therapy could provide additive benefits for cardio-protection, and the mechanisms operating in these processes may be due to ROCK2 diminishment.

\section{Acknowledgments}

Xiao Ke and Bin Ke are co-first authors.

The study was supported by grants from the National Natural Science Foundation of China (81302877) and the Natural Science Foundation of Guangdong Province of China (2016A030313248). 
These two grants provided the budget to carry out the present study.

This is the Science and Technology project of Shenzhen city of China (JCYJ20150402094341897 and JCYJ20170307161535847).

We appreciate very much the help Dr. Congwu Yang provided to us in revising the final version of our paper.

\section{Conflict of interest}

The authors declare no conflict of interest.

\section{References}

1. Goff DC Jr, Lloyd-Jones DM, et al. 2013 ACC/AHA Guideline on the Assessment of Cardiovascular Risk: A Report of the American College of Cardiology/American Heart Association Task Force on Practice Guidelines. Circulation 2013; 24: S74-5.

2. Sattar N, Preiss D, Murray HM, et al. Statins and risk of incident diabetes: a collaborative meta-analysis of randomised statin trials. Lancet 2010; 375: 735-42.

3. Pawelczyk M, Chmielewski H, Kaczorowska B, et al. The influence of statin therapy on platelet activity markers in hyperlipidemic patients after ischemic stroke. Arch Med Sci 2015; 11: 115-21.

4. Ridker PM, Danielson E, Fonseca FA, et al. Rosuvastatin to prevent vascular events in men and women with elevated C-reactive protein. N Engl J Med 2008; 359: 2195-207.

5. Kureishi Y, Luo Z, Shiojima I, et al. The HMG-CoA reductase inhibitor simvastatin activates the protein kinase Akt and promotes angiogenesis in normocholesterolemic animals. Nat Med 2000; 6: 1004-10.

6. Chen Z, Fukutomi T, Zago AC, et al. Simvastatin reduces neointimal thickening in low-density lipoprotein receptor-deficient mice after experimental angioplasty without changing plasma lipids. Circulation 2002; 106: 20-3.

7. Mason RP, Jacob RF, Kubant R, et al. Dipeptidyl peptidase-4 inhibition with saxagliptin enhanced nitric oxide release and reduced blood pressure and sICAM- 1 levels in hypertensive rats. J Cardiovasc Pharmacol 2012; 60: 467-73.

8. Tremblay AJ, Lamarche B, Deacon CF, et al. Effects of sitagliptin therapy on markers of low-grade inflammation and cell adhesion molecules in patients with type 2 diabetes. Metabolism 2014; 63: 1141-8.

9. Dai Y, Dai D, Mercanti F, et al. Dipeptidyl peptidase-4 inhibitors in cardioprotection: a promising therapeutic approach. Acta Diabetol 2013; 50: 827-35.

10. Cai A, Zheng D, Dong Y, et al. Efficacy of atorvastatin combined with adipose-derived mesenchymal stem cell transplantation on cardiac function in rats with acute myocardial infarction. Acta Biochim Biophys Sin (Shanghai) 2011; 43: 857-66.

11. Weissberg PL, Bennett MR. Atherosclerosis: an inflammatory disease. N Engl J Med 1999; 340: 1928-9.

12. Wang L, Qu P, Zhao J, et al. NLRP3 and downstream cytokine expression elevated in the monocytes of patients with coronary artery disease. Arch Med Sci 2014; 10: 791-800.

13. Cai A, Qiu R, Li L, et al. Atorvastatin treatment of rats with ischemia-reperfusion injury improves adipose-de- rived mesenchymal stem cell migration and survival via the SDF-1alpha/CXCR-4 axis. PLoS One 2013; 8: e79100.

14. Stalker TJ, Lefer AM, Scalia R. A new HMG-CoA reductase inhibitor, rosuvastatin, exerts anti-inflammatory effects on the microvascular endothelium: the role of mevalonic acid. Br J Pharmacol 2001; 133: 406-12.

15. Nguyen DCA, Montezano AC, Burger D, et al. Angiotensin II, NADPH oxidase, and redox signaling in the vasculature. Antioxid Redox Signal 2013; 19: 1110-20.

16. Noma $K$, Rikitake $\mathrm{Y}$, Oyama N, et al. ROCK1 mediates leukocyte recruitment and neointima formation following vascular injury. J Clin Invest 2008; 118: 1632-44.

17. Liu L, Liu J, Wong WT, et al. Dipeptidyl peptidase 4 inhibitor sitagliptin protects endothelial function in hypertension through a glucagon-like peptide 1-dependent mechanism. Hypertension 2012; 60: 833-41.

18. Matsubara J, Sugiyama S, Sugamura K, et al. A dipeptidyl peptidase-4 inhibitor, des-fluoro-sitagliptin, improves endothelial function and reduces atherosclerotic lesion formation in apolipoprotein E-deficient mice. J Am Coll Cardiol 2012; 59: 265-76.

19. Matsubara J, Sugiyama S, Akiyama E, et al. Dipeptidyl peptidase-4 inhibitor, sitagliptin, improves endothelial dysfunction in association with its anti-inflammatory effects in patients with coronary artery disease and uncontrolled diabetes. Circ J 2013; 77: 1337-44.

20. Zeng Y, Li C, Guan M, et al. The DPP-4 inhibitor sitagliptin attenuates the progress of atherosclerosis in apolipoprotein-E-knockout mice via AMPK- and MAPK-dependent mechanisms. Cardiovasc Diabetol 2014; 13: 32.

21. Matheeussen V, Waumans Y, Martinet W, et al. Dipeptidyl peptidases in atherosclerosis: expression and role in macrophage differentiation, activation and apoptosis. Basic Res Cardiol 2013; 108: 350 